\title{
Enhancement of Human Cheek Skin Texture by Acacia Nilotica Bark Extract Cream
}

\author{
Atif $\mathrm{Ali}^{1^{\star}}$, Naveed Akhtar ${ }^{1}$ and Haji Muhammad S Khan ${ }^{1}$ \\ Department of Pharmacy, Faculty of Pharmacy and Alternative Medicine, The Islamia University of Bahawalpur, Bahawalpur, \\ Pakistan
}

*For correspondence: Email: ajmaline2000@gmail.com

\begin{abstract}
Purpose: To evaluate the effect of a topical application of a cream formulation containing extract of Acacia nilotica bark extract on human cheek skin texture.

Methods: A cream containing 3 \% concentrated extract of Acacia nilotica bark was developed by entrapping the extract in the internal aqueous phase of the cream having strong antioxidant activity. A similar cream but without the extract was also prepared. Each of the formulations was applied to the cheeks of 11 human volunteers for a period of three months. Both cream formulations were assessed, using Visioscan VC 98, for various parameters including texture parameters (energy, contrast and variance) as well as for skin roughness (Ser), skin scaliness (SEsc), skin smoothness (SEsm) and skin wrinkles (Sew).

Results: Unlike the cream base, the extract cream exhibited steady, significant improvement in skin parameters over the 3-month period of application. Images showed visible improvement of the overall skin appearance and reduction of fine lines by the extract cream. Furthermore, skin texture (variance and contrast) and SELS (SEr, SESC, SEsm and SEw) parameters showed significant decline $(p<0.05)$ and the texture parameter of energy showed significant increase $(p<0.05)$.

Conclusion: Our findings indicate that the cream containing $3 \%$ Acacia nilotica bark extract possesses anti-aging effect and improves skin surface appearance..
\end{abstract}

Keywords: Acacia nilotica, Cream, Visioscan VC 98, Skin texture, Anti-aging

Tropical Journal of Pharmaceutical Research is indexed by Science Citation Index (SciSearch), Scopus, International Pharmaceutical Abstract, Chemical Abstracts, Embase, Index Copernicus, EBSCO, African Index Medicus, JournalSeek, Journal Citation Reports/Science Edition, Directory of Open Access Journals (DOAJ), African Journal Online, Bioline International, Open-J-Gate and Pharmacy Abstracts

\section{INTRODUCTION}

Aging of skin is a composite and unceasing progression that may be remarkably induced by sun exposure [1]. Solar ultraviolet exposure is the main factor that instigates reactive oxygen species (ROS) [2]. ROS can interfere with fatty acids, proteins and saccharides, and results in oxidative impairment [3] leading to collagen insufficiency and, ultimately, skin roughness [4]. Aging is linked with morphological variations [1] and is pigeon-holed by damage of elasticity, increased wrinkling, irregular pigmentation, dryness and roughness [5].

Topical formulations have attracted significant interest as a vehicle for drug delivery to the human skin due to easy compliance [6]. One strategy against skin aging is the use of topical plant phenolics and antioxidants [4].

Acacia nilotica (Mimosaceae) is a remedial plant recognized to contain abundant phenolics, consisting of condensed tannin and gallic acid, phlobatannin, pyrocatechol, (+) -catechin, protocatechuic acid, (-) epi-gallocatechin-7- 
gallate and (-) epigallocatechin-5, 7-digallate and others. Different parts of this plant such as the root, seed, leaf, bark, flower, fruit, gum and immature pod act as anti-mutagenic, strong antioxidant, anti-cancer, vasoconstrictor, spasmogenic, anti-asthamatic, cytotoxic, antipyretic, anti-diabetic, anti-plasmodial and antiplatelet aggregatory agents [7].

The purpose of the present investigation was to evaluate the effect of topical cream application of Acacia nilotica bark on the skin texture of human cheek skin.

\section{EXPERIMENTAL}

\section{Plant material}

The bark of Acacia nilotica was obtained during July in Dera Ghazi Khan, Pakistan. Identification of the plant (Acacia nilotica) was done by a taxonomist, Dr. Muhammad Arshad holding a position at the Cholistan Institute of Desert Studies (CIDS), the Islamia University of Bahawalpur, Bahawalpur, Pakistan. A voucher specimen (no. AN-BK-01-01-10-030) was deposited in the Herbarium, the Islamia University of Bahawalpur.

\section{Other materials}

Abil EM 90 was procured from Franken Chemicals, Germany; paraffin oil from Merck Germany; absolute ethanol from BDH England; and deionized water from the Pharmaceutical Labs of the Department of Pharmacy, The Islamia University of Bahawalpur, Pakistan.

\section{Preparation of extract and determination of free scavenging activity}

The extract of the dried and ground bark of Acacia nilotica was prepared in $80 \%$ aqueous ethanol solution with the aid of a homogenizer (Euro-Star, IKAD 230, Germany). The extract was filtererd through whatman filter paper and then concentrated up to one tenth under reduced pressure at $45{ }^{\circ} \mathrm{C}$ using rotatory evaporator (Eyela, Co. Ltd. Japan). Free scavenging activity of the plant extract alone and of the cream formulations were assessed using the stable free radical, 1, 1-diphenyl-2-picrylhydrazyl (DPPH) assay. In 5 miroleter of aqueous ethanolic plant extract, added DPPH to make the volume up to $100 \mu \mathrm{l}$ in 96-well plates. Mixed the contents and incubated at $37{ }^{\circ} \mathrm{C}$ for $30 \mathrm{~min}$ and measured the optical density at $517 \mathrm{~nm}$. Ascorbic acid was used as the standard.

\section{Preparation of cream formulations}

The extract cream consisted of $14 \%$ paraffin oil, $2 \%$ Abil EM 90, $3 \%$ Acacia nilotica bark extract, $1 \%$ fragrance and deionized water, to $100 \%$. The heated oily and aqueous phases were mixed up to $75 \pm 1^{\circ} \mathrm{C}$ in water bath using a homogenizer (Euro-Star, IKAD 230, Germany), followed by the addition of the plant extract and fragrance. The same method was used to prepare the cream base except that the extract was not incorporated.

\section{Subjects}

Eleven subjects (aged 20 to 35 years) were selected for the study. Helsinki Declaration [17] was followed in this single-blind study. Informed consent was signed by all volunteers before the commencement of the study. All subjects were healthy males with no known dermatological diseases or allergy to substances in the formulations. The exclusion criteria were presence of any dermatitis and/or other skin or allergic diseases, smokers and any previous treatment of cheek skin with cosmetic formulations such as sunscreens, moisturizers or anti-aging cosmetics in the two months prior to the commencement of the study. During the test period, the subjects were allowed to bathe normally, but were instructed not to use any skin care product on their cheeks other than the test formulations.

\section{Instrumental assessment}

Visioscan VC 98 is a special UVA-light video camera with high resolution for the direct study of skin parameters. The image output shows the structure of the skin and the level of dryness. The camera was connected to a computer via a digitalization unit (Video Digitizer VD 300) through FireWire port. All measurements were made in a draught-free room at controlled temperature $\left(18.0-20.6{ }^{\circ} \mathrm{C}\right)$ and relative humidity $(50-65 \%)$.

\section{Study protocol}

In vivo investigations were carried from December 2011 to March 2012. All instrumental measurements were carried out according to manufacturer's instructions. Two weeks before study began and during the treatment period, the volunteers were permitted only the use of normal cleansing products. Each volunteer was then handed two creams - extract cream and base cream which served as control. The volunteers were well-informed about the correct use of the creams. Measurements of skin parameters, 
which included texture parameters (energy, contrast and variance) and for skin roughness (Ser), skin scaliness (SEsc), skin smoothness (SEsm) and skin wrinkles (Sew) were made monthly for 3 months. Approximately $500 \mathrm{mg}$ of extract cream was applied to the right cheek and base cream to the left cheek twice daily (morning 7:00 - 9:00; evening, 19:00 - 21:00) at home by the volunteers. The area around the eyes was omitted. Prior to measurement, the volunteers remained in the room for at least $15 \mathrm{~min}$ in order to allow the skin to adjust to room temperature. , Approval of this study was given by the Board of Advanced Study and Research (BASAR), The Islamia University, Bahawalpur, Pakistan and the Institutional Ethical Committee (ref. no. 3715/Acad), Faculty of Pharmacy and Alternative Medicine, The Islamia University, Bahawalpur.

\section{Statistical analysis}

SPSS 17.0 was used for data analysis by twoway ANOVA to determine differences at various time intervals while paired sample t-test was used to assess differences between the two

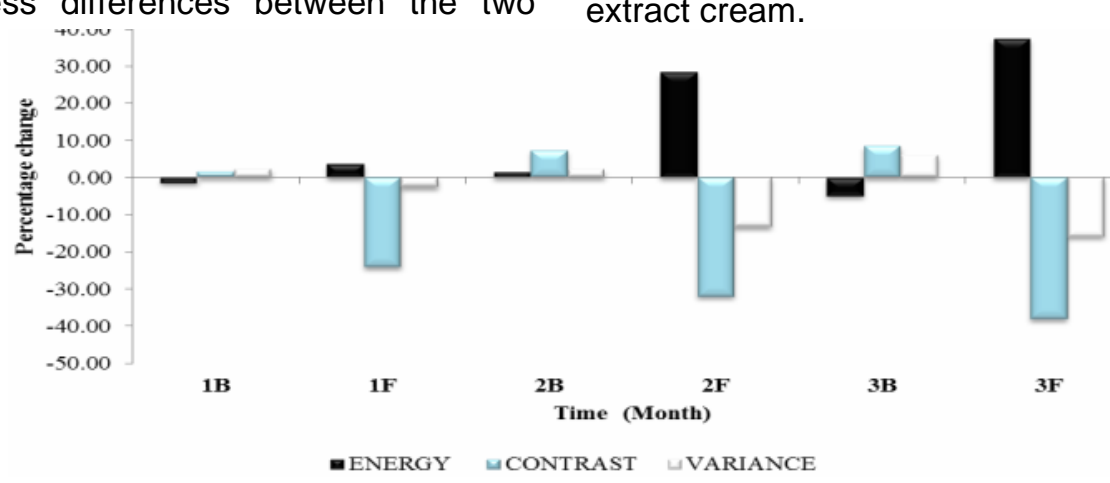

cream formulations. The level of significance was set $p<0.05$.

\section{RESULTS}

The antioxidant activity of plant extract itself and the extract cream was 89 and $83 \%$, respectively. Change in skin texture parameters (energy, contrast and variance) after application of base and extract is shown in Fig 1. Increase in the energy of the extract cream was statistically significant at all time intervals while that of the base cream was insignificant $(p>0.05)$. Similarly, decrease in the variance and contrast of the extract cream was statistically significant at all time intervals but the values for the base cream was insignificant $(p>0.05)$. Significant differences $(p>0.05)$ between the two creams were also found.

SELS data (SEr, SEsc, SEsm and SEw) are shown in Fig 2 while Fig 3 represents the photographs of the right cheek of a human volunteer before and after application of the extract cream.

Fig. 1: Change (\%) in skin texture parameter (energy, contrast and variance) of volunteers with time after following application of base and extract creams. Key: 1B = application of base cream after one month, $1 \mathrm{~F}=$ application of extract cream after one month, $2 \mathrm{~B}$, application of base cream after two months, $2 \mathrm{~F}=$ application of extract cream after two months, 3B = application of base cream after three months, 3F = application of extract cream after three months

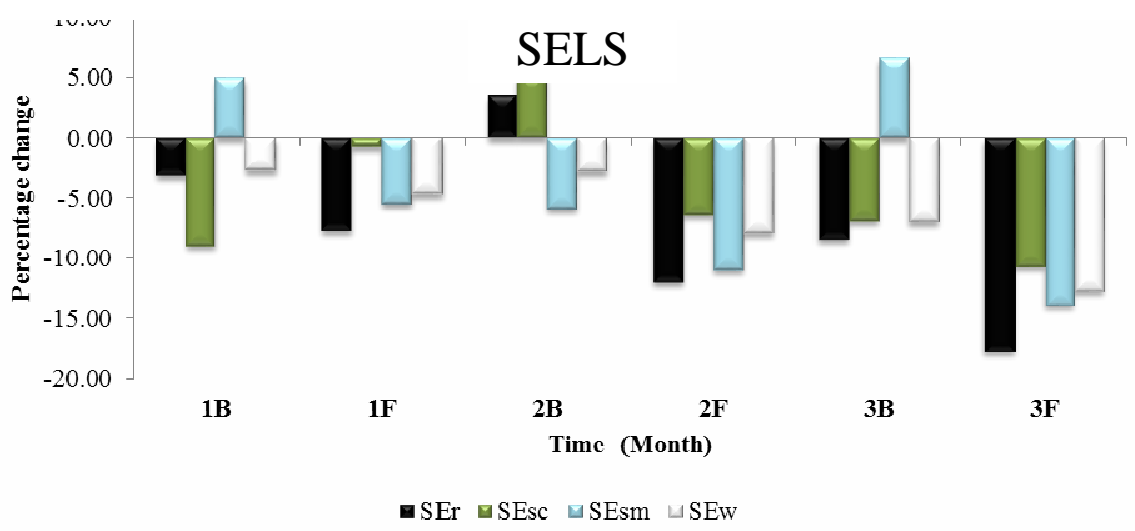

Fig. 2: Change (\%) in SELS parameters of volunteers' skin following application of base and extract creams. Note: $1 \mathrm{~B}=$ application of base cream after one month, $1 \mathrm{~F}=$ application of extract cream after one month, 2B, application of base cream after two months, $2 \mathrm{~F}=$ application of extract cream after two months, $3 \mathrm{~B}=$ application of base cream after three months, $3 \mathrm{~F}=$ application of extract cream after three months. 


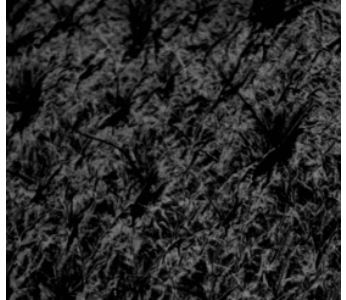

(A)

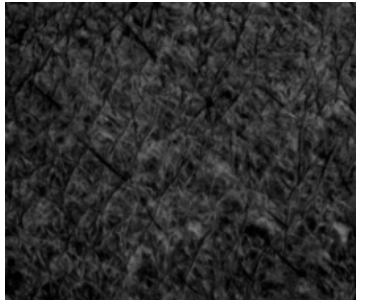

(B)
Fig 3: Photograph of the right cheek of a human volunteer $(A)$ before and $(B)$ after application of extract cream for 3 months The base cream (control) produced statistically insignificant changes in all SELS parameters of the skin but the effect of the extract cream was significant at all time intervals $(p>0.05)$. This difference was also observed when the SELS parameters of the extract and base creams were directly compared $(p>0.05)$.

\section{DISCUSSION}

Phenolic compounds are known to have antioxidant activity and it is likely that the activity of the extract may be due to these compounds [3]. Skin texture parameters (energy, contrast and variance) analyze differences in the colors of neighboring pixels [8]. Energy increases with increase in the hydration level of the skin and this in turn results in greater homogeneity of the output image. Young, highly hydrated, elastic skin has a high energy value compared to older skin with many wrinkles [9]. Increase in energy parameter indicates moisturizing effect of the extract cream and hence anti-aging properties of the extract.

Variance is the average of a local variance over an amount of pixels. The actual value of the pixel is compared to the average. High variance indicates high roughness of skin surface [10]. Our findings indicate reduced roughness of the skin surface.

Contrast indicates the difference between gray levels of the two neighbored pixel. Contrast is higher, the higher the difference between the values of two neighbors [10]. Reduction in contrast indicates better condition and smoothness of skin resulting in a reduction of roughness.

SEr is the roughness parameter which calculates the proportion of dark pixels. SEsm is the index of smoothness and is calculated from the mean width and depth of wrinkles. SEsc is the index of scaliness of skin which shows the level of dryness of the skin. SEw identifies aging including wrinkles and is calculated from the proportion of horizontal and vertical wrinkles [11].
Gradual decrease in the values of roughness, scaliness, smoothness and wrinkles were observed for the extract cream. The extract cream produced a decrease in the mean values of skin smoothness in contrast to skin roughness which suggests that the cream possesses antiaging properties. Smaller SEsc value corresponds to higher skin moisture as treatment with moisturizing or anti-aging formulations results in lower values for SEsc. Lower values for the parameter SEw indicates that there were less wrinkles on the skin indicating that the extract cream reduced fine wrinkles. This is directly related to the loss of collagen which has strong relation with trans-epidermal water loss [12]. Greater epidermal water loss leads to less water retention by collagen and results in collagen degeneration. Elastin is important because it supports the body's natural elasticity. Elastin stops skin from sagging and helps the body repair tissue after a wound. Collagen is a protein that is found in the body's connective tissues [13]. Our findings suggest a reduction in aging characteristics.

Improvement in skin surface parameters can be attributed to the phenolics in the extract of the plant. These phenolics include kaempferol [14], () epicatechin, (+) dicatechin, quercetin, ascorbic acid, gallic acid, (+) leucocyanidin gallate, sucrose and $(+)$ catechin-5-gallate $[7,15]$. Bioflavonoids and polyphenolic compounds in plants have also been shown to demonstrate beneficial antioxidant properties. (-)epigallocatechin-3-gallate (EGCG) is one of such major components and is an effective agent against skin inflammation and carcinogenesis [13]. Phenolics inhibit reactive oxygen species (ROS) and offer the best protection against enzymes that cause the breakdown of collagen and elastin in skin and can help prevent tissue injury $[3,18]$. Also, it has been revealed that ascorbic acid provides photo-protection and prevents inflammation and UVB-induced immune-suppression when applied topically [16]. All of these investigations collectively support our findings that the extract cream possesses antiaging properties.

\section{CONCLUSION}

The findings of this study suggest that the formulated topical cream of Acacia nilotica extract is capable of reducing the signs of skin aging. Further studies are, however, required to unravel the anti-aging mechanism of the plant constituents. 


\section{REFERENCES}

1. Wulf HC, Sandby-Møller J, Kobayasi T, Gniadecki R. Skin aging and natural photoprotection. Micron. 2004; 35: 185-191.

2. González S, Fernández-Lorente M, Gilaberte-Calzada $Y$. The latest on skin photoprotection. Clinic dermatol. 2008; 26: 614-626.

3. Alena S, Jitka Psotova DW. Natural phenolics in the prevention of UV-induced skin damage. A Review. Biomed Papers. 2003; 147: 137-145.

4. Ichihashi, M, M. Ueda, A. Budiyanto, T. Bito, M. Oka, M. Fukunaga, K. Tsuru TH. UV-induced skin damage. Toxicol. 2003; 189: 21-39.

5. Fisher GJ, Kang S, Varani J, Bata-Csorgo Z, Wan Y, Datta $S$, et al. Mechanisms of photoaging and chronological skin aging. Arch Dermatol. 2002; 138: 1462-70.

6. Foldvari $M$. Non-invasive administration of drugs through the skin: challenges in delivery system design. Pharm Sci Technol To. 2000; 3: 417-425.

7. Ali A, Akhtar N, Khan BA, Khan MS, Rasul A, Khalid N, Waseem K, Mahmood T, Ali L. Acacia nilotica: A plant of multipurpose medicinal uses. J Med Plant Res. 2012; 6: 1492-1496.

8. Visioscan® VC 98 and Software SELS (Surface Evaluation of the Living Skin) 2009; 1-53.

9. Rasul A, Akhtar N. Formulation and in vivo evaluation for anti-aging effects of an emulsion containing basil extract using non- invasive biophysical techniques. Daru. 2011; 19: 344-350.

10. Debowska R, Rogiewicz K, Iwanenko T, Kruszewski M, Eris I. Folic Acid (Folacin) - New application of a cosmetic ingredient. Kosmetische Medizin. 2005; 3 . 16-22.
11. Ishii $H$, Kitamura $T$, Todo $H$, Sugibayashi $K$, Sakurai $H$, Fukahori M. Combined effect of sodium chondroitin sulfate and sodium hyaluronate on skin moisturization following single and repeated application. Asian J Pharm Sci. 2008; 3: 94-101.

12. Williams $S$, Tamburic S, Stensvik $H$, Weber M. Changes in skin physiology and clinical appearance after microdroplet placement of hyaluronic acid in aging hands. J Cosmetic Dermatol. 2009; 8: 216-225.

13. Udompataikul $M$, Sripiroj $P$, Palungwachira $P$. An oral nutraceutical containing antioxidants, minerals and glycosaminoglycans improves skin roughness and fine wrinkles. Int J Cosmetic Sci. 2009; 31: 427435.

14. Singh R, Singh B, Singh S, Kumar N, Kumar S, Arora S. Anti-free radical activities of kaempferol isolated from Acacia nilotica (L.) Willd. Ex. Del. Anti-free radical activities of kaempferol isolated from Acacia nilotica (L.) Willd. Ex. Del. Toxicol In Vitro. 2008; 22: $1965-1970$.

15. Agrawal S, Kulkarni GT, Sharma VN. A Comparative Study on the Antioxidant Activity of Methanol Extracts of Acacia. Adv in Nat Appl Sci. 2010; 4: 78-84.

16. Lupo MP. Antioxidants and vitamins in Cosmetics. Clin Dermatol. 2001; 19: 467-473.

17. World Medical Association Declaration of Helsinki. Ethical principles of medical research involving human subjects. [Cited: 2008 September 3] http://www.wma.net/en/30publications/10plicies/b3/ index.html.

18. Nunu M, Louisa N, Ketevan J, Marine D, Eduard C. Identification of free radicals induced by UV irradiation in collagen water solutions. J Photochem Photobiol B. 2008; 93: 61-65. 